九州大学学術情報リポジトリ

Kyushu University Institutional Repository

\title{
BERRY-ESSEEN TYPE BOUND FOR DENSITY ESTIMATORS OF STATIONARY MARKOV PROCESSES
}

Rao, B. L. S. Prakasa

Centre de Recherches Mathematiques, Universite de Montreal

https://doi.org/10.5109/13110

出版情報：統計数理研究. 17 (3/4)，pp. 15-21，1977-03. Research Association of Statistical Sciences

バージョン :

権利関係 : 


\title{
BERRY-ESSEEN TYPE BOUND FOR DENSITY ESTIMATORS OF STATIONARY MARKOV PROCESSES
}

By

\author{
B. L. S. Prakasa RAO* \\ (Received November 1, 1975)
}

\begin{abstract}
Berry-Esseen type bound for the distribution function of a density estimator of kernel type is obtained when the observations are from a stationary Markov process. It is shown that the bound is of the order $0\left(n^{-1 / 3+\tau}\right)$ for any $\tau>0$ under some conditions.
\end{abstract}

\section{Introduction}

Estimators of the density function of a population based on a sample of independent observations have been considered by several authors. An excellent survey of the results in this area is given in Rosenblatt (1971). Rosenblatt (1970) and Roussas $(1967,1969)$ considered kernel type of density estimators when the observations are assumed to be sampled from a stationary Markov process. Rosenblatt (1970) has shown that these estimators have the same character as in the case of independence. He proved that the kernel type estimators are asymptotically normal under some regularity conditions. Our aim in this paper is to obtain Berry-Esseen type bound for the density estimator after proper norming. Result obtained here generalizes a similar result of Wertz (1971) in the independent case. Order of the bound obtained here is the same as in the independent case.

\section{Preliminaries}

Consider a probability space $(R, \mathscr{B}, P)$ and let $\left\{X_{n}, n \geq 1\right\}$ be a Markov process taking values in $(R, \mathcal{B}, P)$ with stationary transition measure $p(\xi, A)=P\left(X_{n+1} \in A \mid X_{n}\right.$ $=\xi)$. Assume that $p(\xi, A)$ is a measurable fuuction of $\xi$ for fixed $A$ and a probability measure on $\mathscr{B}$ for fixed $\xi$. Such a transition measure gives rise to a Markov process by Doob (1953). Assume that the process $\left\{X_{n}, n \geq 1\right\}$ satisfies a Doeblin's condition $\left(D_{0}\right)$ as given by Doob (1953), p. 221 viz. there is a finite-valued measure $\lambda$ on $\mathscr{B}$ with $\lambda(R)>0$, an integer $\nu \geq 1$ and $\varepsilon>0$ such that

AMS (1970) subject classification: Primary 62G05, 62M05. Key words and phrases: Density Estimator, Berry-Esseen type bound, Stationary Markov process.

* Centre de Recherches Mathématiques, Université de Montréal. 


$$
p^{(\nu)}(\xi, A) \leq 1-\varepsilon \text { if } \lambda(A) \leq \varepsilon
$$

and there is only one ergodic set $E$ in $R$ with $\lambda(E)>0$ and this set contains no cyclically moving subsets. (Here $p^{(n)}(\cdot, \cdot)$ is the $n$-step transition measure.) Under $\left(D_{0}\right)$, it can be shown that there exist positive constants $r_{f} \geq 1,0<\rho_{f}<1$ and a unique stationary probability distribution $\Pi(\cdot)$ such that

$$
\left|p^{(n)}(\xi, E)-\Pi(E)\right| \leq r_{f} \rho_{f}^{n}
$$

for $n \geq 1$. The distribution $\Pi(\cdot)$ taken as the initial distribution together with the stochastic transition function determines a stationary Markov Process. We shall assume that the initial distribution is always the stationary distribution. Suppose that $p(\cdot, \cdot)$ and $\Pi(\cdot)$ are absolutely continuous with respect to a $\sigma$-finite measure $\mu$. Let $f(\cdot, \cdot)$ and $f(\cdot)$ be densities of $p(\cdot, \cdot)$ and $\Pi(\cdot)$ with respect to $\mu$. The problem which we consider in this paper is to find the rate of convergence of distribution of kernel type density estimators of $f(\cdot)$. Let $P_{f}$ be the probability measure on $\left(R^{\infty}, \mathscr{B}^{\infty}\right)$ corresponding to $f(\cdot, \cdot)$ and $f(\cdot)$. Here after, we shall suppose that $R$ is the real line and $\mathscr{B}$ the $\sigma$-field of Borel sets of $R, \mu$ Lebesgue measure.

We shall now state a Berry-Esseen type theorem for random variables defined on a Markov process satisfying the above conditions.

THEOREM 2.1. Let $Z_{j_{n}}=a_{n} M\left(b_{n} X_{j}+c_{n}\right), 1 \leq j \leq n, n \geq 1$ be a double sequence of random variables defined on a stationary Markov process $\left\{X_{n}, n \geq 1\right\}$ as given above where $\left\{a_{n}\right\}$, $\left\{b_{n}\right\}$ and $\left\{c_{n}\right\}$ are sequences of non-negative constants such that $a_{n} \rightarrow 0$ and $M(\cdot)$ is $a$ bounded real valued function. Further suppose that $\mathrm{E}\left(Z_{j n}\right)=0$ for all $j$ and $n$. Define

$$
\begin{aligned}
& \beta_{i n}=\mathrm{E}\left|Z_{1 n}\right|^{i}, \mathrm{n} \geq 1 \\
& \sigma_{n}^{2}=n^{-1} \operatorname{Var}\left[\sum_{j=1}^{n} Z_{j n}\right] .
\end{aligned}
$$

Suppose that $\sigma_{n}^{2}>0$. Let

$$
S_{n}=n^{-1 / 2} \sigma_{n}{ }^{1} \sum_{j=1}^{n} Z_{j n}
$$

Then there exist absolute constants $C_{i}, i=1,2,3$ such that for every $n$,

$$
\begin{aligned}
\sup _{-\infty<u<+\infty} \mid & P\left(S_{n} \leq u\right)-\Phi(u) \mid \\
& \leqq C_{1} n^{-1 / 2} \frac{\beta_{3 n}}{\sigma_{n}^{3}}\left\{1+\left[\frac{r_{f}^{2}}{1-\rho_{f}}\right]^{2}\right\} \\
& +C_{2} n^{-1 / 2} \frac{\beta_{1 n}}{\sigma_{n}}\left[\frac{r_{f}^{2}}{1-\rho_{f}}\right] \\
& +C_{3}\left[\frac{\rho+\sqrt{ } 2 r_{f}}{1+\sqrt{ } 2 r_{f}}\right]^{n} \frac{\beta_{1 n} \sigma_{n}^{2}}{\beta_{3 n}} \frac{r_{f}^{2}}{\left(1-\rho_{f}\right)^{2}}
\end{aligned}
$$

where $\Phi$ is the standard normal distribution.

Lemma 3.4 of Prakasa Rao (1973) is similar to this theorem and it is based on a result of Aleskevicus (1966). We omit the oroof this result. We shall now state a lemma due to Wertz (1971) which will be used in the sequel.

LeMMA 2.1. Let $a, b$ and $c$ be positive real numbers, $\left(\Omega_{n}, \mathcal{B}_{n}, P_{n}\right), n \geq 1$ be a sequence of probability spaces, and suppose that $\left\{\zeta_{n}\right\}$ and $\left\{\eta_{n}\right\}$ are sequences of real valued 
random variables defined on $\left(\Omega_{n}, \mathscr{B}_{n}, P_{n}\right)$. Further suppose that there exists positive constants $C, C^{\prime}$ such that for every $y \in R$ and for every $n \geq 1$,

$$
\begin{aligned}
& \left|P_{n}\left(\eta_{n} \leq y\right)-\Phi(y)\right| \leq C n^{-c}, \\
& P_{n}\left(\left|\zeta_{n}\right| \geq n^{-a}\right) \leq C^{\prime} n^{-b} .
\end{aligned}
$$

Then there exists $C^{\prime \prime}>0$ such that for every $y \in R$ and for every $n \geq 1$

$$
\left|P_{n}\left(\zeta_{n}+\eta_{n} \leq y\right)-\Phi(y)\right| \leq C^{\prime \prime} n^{-\min (a, b, c)} .
$$

We shall state few more lemmas which give upper and lower bounds for the variance of sums of random variables $f\left(X_{j}\right)$ when $\left\{X_{j}\right\}$ is a stationary Markov process satisfying Doeblin's condition $\left(D_{0}\right)$.

Lemia 2. 2. (Doob (1953), p. 222). Let $g(\bullet)$ be $\mathcal{B}$-measurable with $\mathrm{E}\left[g\left(X_{1}\right)\right]^{2}<\infty$. Then

$$
\begin{aligned}
\mid \mathrm{E}\left(g\left(X_{1}\right) g\left(X_{k+1}\right)\right) & -\mathrm{E}\left(g\left(X_{1}\right)\right) \mathrm{E}\left(g\left(X_{k+1}\right)\right) \mid \\
& \leq 2 r_{f}^{1 / 2} \rho_{j}^{k / 2} \mathrm{E}\left[\mathrm{g}\left(X_{1}\right)\right]^{2} .
\end{aligned}
$$

LEMma 2.3. (Prakasa Rao (1973), p. 144). Let $g(\cdot)$ be $\mathscr{B}$-measurable with $\sigma^{2}=$ $E\left[g\left(X_{1}\right)\right]^{2}<\infty$. Then

$$
\operatorname{Var}\left[\sum_{i=1}^{n} g\left(X_{i}\right)\right] \leq n \sigma^{2}\left\{1+4 r_{f}^{1 / 2}\left(1-\rho_{f}^{1 / 2}\right)^{-1}\right\} .
$$

Lemma 2.4. Let $g(\cdot)$ be $\mathscr{B}$-measurable with $\mathrm{E}\left[g\left(X_{1}\right)\right]=0, \sigma^{2}=\mathrm{E}\left[g\left(X_{1}\right)\right]^{2}<\infty$. Then

$$
\operatorname{Var}\left[\sum_{i=1}^{n} g\left(X_{i}\right)\right] \geq n \sigma^{2}\left\{1-\rho_{f}^{1 / 2}\left(1+4 r_{f}^{1 / 2}\right)\right\}\left(1-\rho_{f}^{1 / 2}\right)^{-1} .
$$

Proof: By the stationarity of the process $\left\{X_{j}\right\}$ and the fact that $\mathrm{E}\left[g\left(X_{j}\right)\right]=0$,

$$
\begin{aligned}
\operatorname{Var}\left[\sum_{i=1}^{n} g\left(X_{i}\right)\right]=\mathrm{E}\left[\sum_{i=1}^{n} g\left(X_{i}\right)\right]^{2} \\
=n \sigma^{2}+2 \sum_{k=1}^{n-1}(n-k) \mathrm{E}\left[g\left(X_{1}\right) g\left(X_{k+1}\right)\right] \\
\geq n \sigma^{2}-4 r_{f}^{1 / 2} \sigma^{2} \sum_{k=1}^{n-1}(n-k) \rho_{f}^{k / 2} \quad \text { By Lemma 2.2) } \\
=n \sigma^{2}\left\{1-4 r_{f}^{1 / 2} \sum_{k=1}^{n-1} \frac{n-k}{n} \rho_{f}^{k / 2}\right\} \\
\geq n \sigma^{2}\left\{1-4 r_{f}^{1 / 2} \sum_{k=1}^{n-1} \rho_{f}^{k / 2}\right\} \\
=n \sigma^{2}\left\{1-4 r_{f}^{1 / 2} \frac{\rho_{f}^{1 / 2}\left(1-\rho_{f}^{\frac{n-1}{2}}\right)}{1-\rho_{f}^{1 / 2}}\right\} \\
\geq n \sigma^{2}\left\{1-\frac{4 r_{f}^{1 / 2} \rho_{f}^{1 / 2}}{\left.1-\rho_{f}^{1 / 2}\right\}}\right. \\
=n \sigma^{2}\left\{1-\rho_{f}^{1 / 2}\left(1+4 r_{f}^{1 / 2}\right)\right\}\left(1-\rho_{f}^{1 / 2}\right)^{-1} .
\end{aligned}
$$




\section{Main Result}

Let $\left\{X_{n}, n \geq 1\right\}$ be a stationary Markov process satisfying Doeblin's condition $\left(D_{0}\right)$ and other assumptions at the begining of section 2 . Let $f$ denote the density of $X_{j}$ and $r_{f}$ and $\rho_{f}$ be the Doeblin's constants in (2.2).

Let $I$ be any interval on $R$ and $0<\varepsilon_{1}<\varepsilon_{2}, A>0, r \geq 1,0<\rho, h, q<1$. Let $F_{0}=F_{0}$ $\left(A, \rho, h, q, r ; I, \varepsilon_{1}, \varepsilon_{2}\right)$ be the class of all stationary Markov processes with marginal density $f$ and the Doeblin's constants $r_{f}$ and $\rho_{f}$ satisfynig the following conditions:

$$
\begin{array}{ll}
\text { (i) } & 0<\varepsilon_{1} \leq \inf _{x \in I} f(x) \leq \sup _{x \in I} f(x) \leq \varepsilon_{2}, \\
\text { (ii) } & f^{\prime \prime}(x) \text { exists for every } x \in R \text { and } \sup \left\{\left|f^{\prime \prime}(x)\right|: x \in R\right\} \leq A<\propto, \\
\text { (iii) } & 1 \leq r_{f} \leq r<\infty, 0<\rho_{f} \leq \rho<1, \\
& 0 \leq\left(\rho_{f}+2^{1 / 2} r_{f}^{1 / 2}\right)\left(1+2^{1 / 2} r_{f}^{1 / 2}\right)^{-1} \leq h<1 \text {, and } \\
& 0 \leq \rho_{f}^{1 / 2}\left(1+4 r_{f}^{1 / 2}\right) \leq q<1 .
\end{array}
$$

Let $K(\cdot)$ be any bounded continuous symmetric density function such that $c_{i, k}<\infty$ for $0 \leq i \leq 3,0 \leq k \leq 2$ where

$$
c_{i, k}=\int_{R} t^{k} K^{i}(t) d t .
$$

Let $k_{n} \rightarrow \infty$ as $n \rightarrow \infty$ such that $n k_{n} \rightarrow \infty$ as $n \rightarrow \infty$. Define

$$
h_{n}\left(X^{n} ; x\right)=\frac{1}{n k_{n}} \sum_{i=1}^{n} k\left[\frac{x-X_{i}}{k_{n}}\right],\left(x^{n}, x\right) \in R^{n} \times R .
$$

Rosenblatt (1970) proved that

$$
\sqrt{n k_{n}}\left[h_{n}\left(X^{n} ; x\right)-\mathrm{E}\left(h_{n}\left(X^{n} ; x\right)\right)\right]
$$

is asymptotically normal with mean 0 and variance

$$
f(x) \int_{-\infty}^{\infty} K^{2}(t) d t=f(x) c_{2,0}
$$

under some conditions. Let $\Delta_{n}^{2}(f ; x)=\operatorname{Var}\left(h_{n}\left(X^{n} ; x\right)\right)$.

We shall now state and prove the main theorem of this paper.

THEOREM 3.1. Let $k_{n}=n^{-1 / 3}$. Given any $0<\tau<\frac{1}{3}$, there exists $n_{0}$ depending on $F_{0}$ such that

$$
\sup _{F 0} \sup _{x \in I} \sup _{n \geqq n} \sup _{y \in R} n^{1 / 3-r}\left|P_{f}\left[\frac{h_{n}\left(X^{n} ; x\right)-f(x)}{\Delta_{n}(f ; x)} \leq y\right]-\Phi(y)\right|<\infty .
$$

Proof: Let

$$
\eta_{j n}=\frac{1}{n k_{n}} K\left[\frac{x-X_{j}}{k_{n}}\right], 1 \leq j \leq n .
$$

Since $\left\{X_{j}\right\}$ is stationary, $\eta_{j n}, 1 \leq j \leq n$ are identically distributed. Let

$$
\sigma_{n}^{2}=\sigma_{n}^{2}(f ; x)=\operatorname{Var}_{f}\left[\eta_{j n}\right] .
$$

Lemma 2.3 implies that

$$
\operatorname{Var}_{f}\left[\sum_{j=1}^{n} \eta_{j n}\right] \leq n \sigma_{n}^{2}\left[1+4 r_{f}^{1 / 2}\left(1-\rho_{f}^{1 / 2}\right)^{-1}\right]
$$


Hence

$$
\begin{aligned}
\operatorname{Var}_{f}\left[h_{n}\left(X^{n} ; x\right)\right] & \equiv \Delta_{n}^{2}(f ; x) \\
& =0\left(n \sigma_{n}^{2}\right)
\end{aligned}
$$

uniformly in $F_{0}$. Let $\nu_{n i}(f ; x)=\mathrm{E}_{f}\left|\eta_{j n}-E\left(\eta_{j n}\right)\right|^{i}$ and

$$
Z_{j n}=\eta_{j n}-E\left(\eta_{j n}\right), 1 \leqq j \leqq n .
$$

Equations (10) and (11) of Wertz (1971) show that there exists an integer $N_{1}$ depending only on $F_{0}$ such that

$$
n^{-3 / 2} \sigma_{n}^{-3}(f ; x)=0\left(\left(n k_{n}\right)^{3 / 2}\right)
$$

and

$$
\nu_{n 3}(f ; x) \leqq \frac{f(x) c_{3,0}}{n^{3} k_{n}^{2}}+\frac{C_{4}}{n^{3} k_{n}}
$$

for every $n>N_{1}$, uniformly for $F_{0}$ and $x \in I$ where $C_{4}$ is again a constant depending only on $F_{0}$. Applying Theorem 2.1, we obtain that there exists absolute constants $C_{i}, 1 \leqq i \leqq 3$ such that

$$
\begin{aligned}
& \sup _{y \in R}\left|P_{f}\left[\frac{\sum_{j=1}^{n} Z_{j n}}{\Delta_{n}(f ; x)}\right]-\Phi(y)\right| \\
& \quad \leqq C_{1} n^{-1 / 2} \frac{\nu_{n 3}(f ; x)}{\left(n^{-1 / 2} \Delta_{n}(f ; x)\right)^{3}}\left\{1+\left[\frac{r_{f}^{2}}{1-\rho_{f}}\right]^{2}\right\} \\
&+C_{2} n^{-1 / 2} \frac{\nu_{n 1}(f ; x)}{n^{-1 / 2} \Delta_{n}(f ; x)}\left[\frac{r_{f}^{2}}{\rho_{f}}\right] \\
&+C_{3} \frac{\nu_{n 1}(f ; x)\left(n^{-1 / 2} \Delta_{n}(f ; x)\right)^{2}}{\nu_{n 3}(f ; x)}\left[\frac{\rho_{f}+\sqrt{2 r_{f}}}{1+\sqrt{2 r_{f}}}\right]^{n} \frac{r_{f}^{2}}{\left(1-\rho_{f}\right)^{2}} \\
& \leqq \\
& C_{1} n\left\{\frac{f(x) c_{3,0}}{n^{3} k_{n}^{2}}+\frac{C_{4}}{n^{3} k_{n}}\right\} \Delta_{n}^{-3}(f ; x)\left\{1+r^{4}(1-\rho)^{-2}\right\} \\
&+C_{2}\left\{\frac{f(x) c_{3,0}}{n^{3} k_{n}^{2}}+\frac{C_{4}}{n^{3} k_{n}}\right\}^{1 / 3} \Delta_{n}^{-1}(f ; x)\left\{r^{2}(1-\rho)^{-1}\right\} \\
&+C_{3} n^{-1}\left\{\frac{f(x) c_{3,0}}{n^{3} k_{n}^{2}}+\frac{C_{4}}{n^{3} k_{n}}\right\}^{1 / 3} \frac{\Delta_{n}^{2}(f ; x)}{\nu_{n 3}(f ; x)} h^{n} r^{2}(1-\rho)^{-2} .
\end{aligned}
$$

The last inequality follows from the fact that $\nu_{n 1}^{3} \leqq \nu_{n 3}$. Equation (13) of Wertz (1971) shows that there exists $N_{2}$ depending on $F_{0}$ and a constant $C_{5}$ depending on $F_{0}$ such that

$$
n^{-1 / 2} \sigma_{n}^{-1}(f ; x) \leqq C_{5}\left(n k_{n}\right)^{1 / 2}
$$

for all $n>N_{2}$, uniformly for $F_{0}$ and $x \in I$. Lemma 2.4 implies that

$$
\begin{aligned}
\Delta_{n}^{2}(f ; x) & \geqq C_{6} n \sigma_{n}^{2}(f ; x) \\
& \geqq C_{6} C_{5}\left(n k_{n}\right)^{-1}
\end{aligned}
$$

for some $C_{6}$ depending on $F_{0}$. (3.12), (3.14) and (3.16) together prove that R.H.S. of (3.14) is less than or equal to 


$$
\begin{aligned}
& C_{7}\left(n k_{n}\right)^{-1 / 2} \\
+ & C_{8}\left(n k_{n}\right)^{-1 / 2} k_{n}^{1 / 3} \\
+ & C_{9}\left(n k_{n}\right)^{-1 / 2} n^{-3 / 2} k_{n}^{-1 / 6} h^{n} \Delta_{n}^{2}(f ; x) \nu_{n}^{-1}(f ; x)
\end{aligned}
$$

where $C_{7}, C_{8}$ and $C_{9}$ are constants depending only on $F_{0}$. Note that, by Lemma 2.3,

$$
\mathcal{J}_{n}^{2}(f ; x) \leqq C_{10} n \sigma_{n}^{2}(f ; x)
$$

where $C_{10}$ depends only on $F_{0}$ and

$$
\sigma_{n}^{2}(f ; x) \leqq C_{11} n^{-2} k_{n}^{-2} \text {. }
$$

Hence

$$
\Delta_{n}^{2}(f ; x) \leqq C_{10} C_{11} n^{-1} k_{n}^{-2}
$$

Furthermore

$$
\begin{aligned}
\mathcal{\nu}_{n 3}(f ; x) & \geqq\left[\sigma_{n}^{2}(f ; x)\right]^{3 / 2} \\
& \geqq\left[C_{5}^{-2} n^{-2} k_{n}^{-1}\right]^{3 / 2}=C_{12} n^{-3} k_{n}^{-3 / 2}
\end{aligned}
$$

where $C_{12}$ depends only on $\mathrm{F}_{0}$ by $(3.15)^{\cdot}$ Combining (3.20) and (3.21), it follows that

$$
\begin{aligned}
\Delta_{n}^{2}(f ; x) & \nu_{n 3}^{-1}(f ; x) \\
& \leqq C_{10} C_{11} C_{12}^{-1} n^{3} k_{n}^{3 / 2} \\
& =C_{13} n^{2} k_{n}^{-1 / 2}
\end{aligned}
$$

wwhere $C_{13}$ depends only on $F_{0}$. Observe that the last term in (3.17) is still $0\left(\left(n k_{n}\right)^{-1 / 2}\right)$ since $h^{n}$ with $0<h<1$ is involved in it. (3.17) proves that there exists a constant $C_{14}$ depending only on $F_{0}$ such that

$$
\sup _{y \in R}\left|P_{f}\left[\frac{\sum_{j=1}^{n} Z_{j n}}{\Delta_{n}(f ; x)} \leqq y\right]-\Phi(y)\right| \leqq C_{14}\left(n k_{n}\right)^{-1 / 2}
$$

uniformly for $F_{0}$ and $x \in I$ and $n>N_{3}$ depending only on $F_{0}$. Note that $\sum_{j=1}^{n} Z_{j n}$ $=h_{n}\left(X^{n} ; x\right)-\mathrm{E}_{f}\left[h_{n}\left(X^{n} ; x\right)\right]$. Hence

$$
\sup _{y \in R}\left|P_{f}\left[\frac{h_{n}\left(X^{n} ; x\right)-E_{f}\left[h_{n}\left(X^{n} ; x\right)\right]}{\Delta_{n}(f ; x)} \leqq y\right]-\Phi(y)\right| \leqq C_{14}\left(n k_{n}\right)^{-1 / 2}
$$

uniformly for $F_{0}, x \in I$ and $n>N_{3}$ depending only on $F_{0}$.

Let

$$
\eta_{n}=\frac{h_{n}\left(X^{n} ; x\right)-\mathrm{E}_{f}\left[h_{n}\left(X^{n} ; x\right)\right]}{\Delta_{n}(f ; x)}
$$

and

$$
\zeta_{n}=\frac{E_{f}\left[h_{n}\left(X^{n} ; x\right)\right]-f(x)}{\Delta_{n}(f ; x)} .
$$

Note that

$$
\begin{aligned}
& \left|\mathrm{E}_{f}\left[h_{n}\left(X^{n} ; x\right)\right]-f(x)\right| \\
& \quad=\left|\int_{R} K(t) f\left(x+k_{n} t\right) d t-f(x)\right|
\end{aligned}
$$




$$
\leqq 1 / 2 k_{n}^{2}\left\|f^{\prime \prime}\right\|_{\infty} c_{1,2}
$$

where $\left\|f^{\prime \prime}\right\|_{\infty}=\sup \left\{\left|f^{\prime \prime}(x)\right|: x \in R\right\}$ and

$$
\Delta_{n}(f ; x) \geqq\left(C_{6} C_{5}\right)^{1 / 2}\left(n k_{n}\right)^{-1 / 2}
$$

by (3.16). Hence

$$
\left|\zeta_{n}\right| \leqq C_{15}\left(n k_{n}^{5}\right)^{1 / 2}
$$

for $n>N_{4}$ depending on $F_{0}$ and for some constant $C_{15}$ depending on $F_{0}$. Hence, by Tchebyshev's inequality, for any $a>0$ and $p>0$ and $k_{n}=n^{-\alpha}$,

$$
\begin{aligned}
P_{f}\left(\left|\zeta_{n}\right| \geqq n^{-a}\right) & \leqq n^{a p} \mathrm{E}_{f}\left|\zeta_{n}\right|^{p} \\
& \leqq C_{15}^{p} n^{a p}\left(n k_{n}^{5}\right)^{p / 2} \\
& =C_{15}^{p} n^{-p(5 \alpha-2 a-1) / 2}
\end{aligned}
$$

uniformly for $F_{0}$ and $x \in I$. (3.24) shows that for $n>N_{3}$ depending on $F_{0}$

$$
\sup _{F 0} \sup _{x \in I} \sup _{y \in R}\left|\mathrm{P}_{f}\left(\eta_{n} \leqq y\right)-\Phi(y)\right| \leqq C_{14} n^{-(1-\alpha) / 2} .
$$

(3.28) and (3.29) hold for $n>N$ depending on $F_{0}$. Let $a=a, b=\frac{p}{2}(5 \alpha-2 a-1), p>\frac{1}{3 \tau}-1$ and $c=(1-\alpha) / 2$ and choose $\alpha$ such that $5 \alpha-2 a-1>0$ and $0<\alpha<1$. In particular, choosing $a=\frac{1}{3}-\tau$ for any $\tau<\frac{1}{3}$, and applying Lemma 2.1, we obtain (3.5).

REMARK: The result can be extended to estimators of two-dimensional density of the stationary Markov process in an analogous manner.

\section{Ref erences}

[1] Aleskevicus, G. J. Some limit theorems for random variables defined on a homogeneous regular Markov chain. Litovsk. Math. Sb. 6 (1966), 297-311. (In Russian.)

[ 2 ] DоOB, J.L. Stochastic Processes. Wiley, New York, 1953.

[ 3 ] PRAKASA RaO, B.L.S. On the rate of convergence of estimators for Markov processes. Z. Wahrscheinlichkeitstheorie und Verw. Gebiete. 26 (1973), 141-152.

[ 4 ] Rosenblatt, M. Density estimates anc Markov sequences. In Nonparametric Techniques in Statistical Inference (ed. Madan Lal Puri). Cambridge Univ. Press (1970).

[ 5 ] Rosenblatt, M. Curve Estimates. Ann. Math. Statist. 42 (1971), 1815-1842.

[ 6 ] Roussas, G. G. Nonparametric estimation in Markov processes. Technibal Report No. 110. Department of Statistics, Univ. of Wisconsin, Madison, Wisconin (1967).

[ 7 ] Roussas, G.G. Nonparametric estimation of the transition distribution function of a Markov process. Ann. Math. Statist. 40 (1969), 1386-1400.

[8] WeRTz, W. Empirische betrachtungen und normalapproximation bei dichteschätzungen. Operations Research-Verfahren, 8 (1971), 430-448.

B. L.S. Prakasa Rao

Indian Statistical Institute

7, S. J.S. Sansanwal Marg

New Delhi 110029

India 